\title{
Does the Location and Prevelance of Gastric Intestinal Metaplasia Change in Patients with Functional Dyspepsia
}

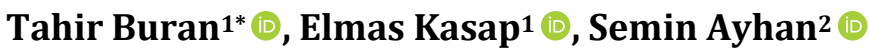 \\ ${ }^{1}$ Department of Gastroenterology, Manisa Celal Bayar University Faculty of Medicine, Manisa, Turkey \\ ${ }^{2}$ Department of Pathology, Manisa Celal Bayar University Faculty of Medicine, Manisa, Turkey \\ Email: ^tahir.buran@hotmail.com, elmaskasap@gmail.com, seminayhan@gmail.com
}

How to cite this paper: Buran, T., Kasap, E. and Ayhan, S. (2021) Does the Location and Prevelance of Gastric Intestinal Metaplasia Change in Patients with Functional Dyspepsia. Open Access Library Journal, 8: e7982.

https://doi.org/10.4236/oalib.1107982

Received: September 19, 2021

Accepted: December 12, 2021

Published: December 15, 2021

Copyright $\odot 2021$ by author(s) and Open Access Library Inc.

This work is licensed under the Creative Commons Attribution International License (CC BY 4.0).

http://creativecommons.org/licenses/by/4.0/

(c) (i) Open Access

\begin{abstract}
Gastric intestinal metaplasia plays a significant role in the pathogenesis of intestinal gastric cancer. Objective: A retrospective analysis of demographic characteristics, site of involvement, and incidence was performed involving patients who underwent endoscopy who had biopsy-diagnosed intestinal metaplasia. Materials and Methods: The study included 720 patients with functional dyspepsia retrospectively from among 6160 patients who underwent upper endoscopy in Celal Bayar University, Faculty of Medicine, Gastroenterology Department, between 2014 and 2020. Results: Intestinal metaplasia was detected at the antrum, corpus, and angulus in $59.94 \%, 13.47 \%$, and $28.57 \%$ of the cases, respectively. Antral lesions were significantly more prevalent $(\mathrm{P}<$ 0.05). Of the patients with IM, $63.5 \%$ had HP positivity, with a significant association between IM positivity and HP positivity $(\mathrm{P}<0.05)$. There was a significant difference between patients with or without a history of chronic PPI use in terms of the prevalence of intestinal metaplasia $(\mathrm{P}<0.05)$. Conclusion: These results suggest that the endoscopic follow-up of patients with IM requires additional attention to the lesser curvature of the antrum as well as the gastric angulus.
\end{abstract}

\section{Subject Areas \\ Biochemistry \\ Keywords \\ Gastric İntestinal Metaplasia, Helicobacter Pylori, Gastritis, Functional Dispepsia}




\section{Introduction}

Gastric cancer is a major public health problem that is the fifth most frequent cancer type and the third leading cause of cancer-related deaths globally [1]. The acknowledged gastric carcinogenesis cascade proposed by Correa is considered initiated by Helicobacter pylori (HP) infection, followed by stages of superficial gastritis, chronic atrophic gastritis, intestinal metaplasia (IM), dysplasia, and gastric cancer [2]. Herein, IM represents an intermediate step that may lead to the development of intestinal type gastric adenocarcinoma.

In 1994, HP was declared a primary carcinogen by the World Health Organization. Chronic HP infection is known to be closely related to the development of atrophic gastritis and IM. Thus, the role of HP treatment in the carcinogenesis cascade remains an active area of research, particularly in terms of the potential reversibility of atrophy and metaplasia.

IM plays a major role in the pathogenesis of intestinal-type gastric cancer as suggested by studies such as those of Gomez et al. that showed a six-fold increased risk of gastric cancer in patients with IM compared to the general population [3] [4].

Thus, the presence of IM is an obvious risk factor for the development of gastric cancer, and early identification of IM represents the single most important step in the follow-up of patients with a risk of gastric cancer [5]. Previous studies have shown that eradication of HP may prevent the development of gastric cancer by alleviating atrophic gastritis [6]. Furthermore, a meta-analysis found that oxyntic atrophy plays a role in the pathogenesis of gastric cancer [7] [8].

Several recent studies also reported that long-term use of PPIs may be a risk factor for the pathogenesis of gastric cancer [9]. It has been proposed that the suppression of gastric acidity may lead to hypergastrinemia via a negative feedback mechanism, resulting in an increased risk of gastric cancer [8]. Additionally, investigations into the role of gastric metaplasia in the pathogenesis of gastric cancer suggested that it may occur as a long-term consequence of atrophic gastritis [10].

Gastric cancer screening is associated with reduced mortality by allowing timely diagnosis and treatment of the condition [11].

Patients with chronic atrophic gastritis or IM have an increased risk of gastric adenocarcinoma. Since atrophy and IM exhibit an irregular distribution pattern in the stomach, the more recently published guidelines, an international biopsy protocol has been developed to detect atrophic and metaplasic changes by obtaining 2 antral (one from the lesser and greater curvature), 2 corpus, and 1 incisura angularis samples. The antral biopsy samples should be obtained $2-3 \mathrm{~cm}$ from the pylorus, while corpus biopsy samples should be $8 \mathrm{~cm}$ from the cardia. In the presence of additional lesions, target biopsies should also be performed [2]. Regular follow-up is not recommended if limited (mild or moderate) atrophy is present. However, in patients with IM confined to a single area who have a family history of gastric cancer, incomplete IM or Helicobacter pylori infec- 
tion, endoscopic follow-up with chromo-endoscopy and biopsy is recommended. Endoscopic follow-up examinations every three years are also recommended for patients with severe atrophic gastritis.

HP eradication may improve atrophic gastritis and reduce the risk of gastric cancer. However, in patients with intestinal metaplasia, HP eradication does not alleviate intestinal metaplasia, although it may slow down the process of neoplastic transformation. Therefore, HP eradication is recommended [2].

In this retrospective analysis, patients undergoing endoscopy due to dyspeptic complaints who were found to have biopsy-diagnosed IM were compared to patients without this condition in terms of demographic characteristics, site of involvement, and incidence. Furthermore, patients with longer-term ( $\geq 3$ months) use of PPIs were compared with no such use of PPIs regarding intestinal metaplasia. Additionally, the association between HP positivity and IM was investigated using the most recent Sydney grading system.

This study was conducted on 720 patients whose biopsies were taken according to Sydney classification by scanning 6120 patients and diagnosed by the same endoscopist and pathologist.

\section{Materials and Methods}

The study included 720 patients with functional dyspeptia retrospectively from among 6160 patients who underwent upper endoscopy in Celal Bayar University, Faculty of Medicine, Gastroenterology Department, between January 2014 and April 2020.

Of these, 5540 subjects were excluded due to reasons previous history of esophageal or gastric surgery, diagnosis of gastric cancer, alarm symptoms, HP eradication treatment within the past 2 months, use of antibiotics, and inadequate number (i.e. $\geq 5$ ) of sampling sites (Figure 1).

Signed consent was obtained from all subjects in the endoscopy unit before the endoscopic procedure. After 8 - 12 hours of fasting, local oropharyngeal sedation was performed using $2 \%$ Xylocaine spray, and intravenous midazolam $(0.07-0.1 \mathrm{mg} / \mathrm{kg})$ was administered immediately prior to the procedure. A gastroscope with an endoscopic video information system was used in the process of the esophagogastroduodenoscopy (EGD) (NBI system using video endoscopes [GIF-H260; Olympus], video processor [Evis Lucera CV 260 SL; Olympus], and lighting unit [Evis Lucerna CLV 260 SL; Olympus]).

A total of 5 gastric biopsy samples were from the following sites: antrum anterior wall, antrum posterior wall, corpus anterior wall, corpus posterior wall, and gastric angulus. Biopsy materials were fixed in $10 \%$ formaldehyde solution and were sent to the pathology laboratory, where tissue sections with 4 to $6 \mu \mathrm{m}$ thickness were prepared from paraffin blocks and stained with hematoxylin and eosin. Then the presence of HP was examined histologically, and the samples were assessed by experienced gastroenterology pathologists to check for gastric atrophy and intestinal metaplasia using the Sydney grading system. HP positivity 
6160 patients screened for eligibility based on medical records

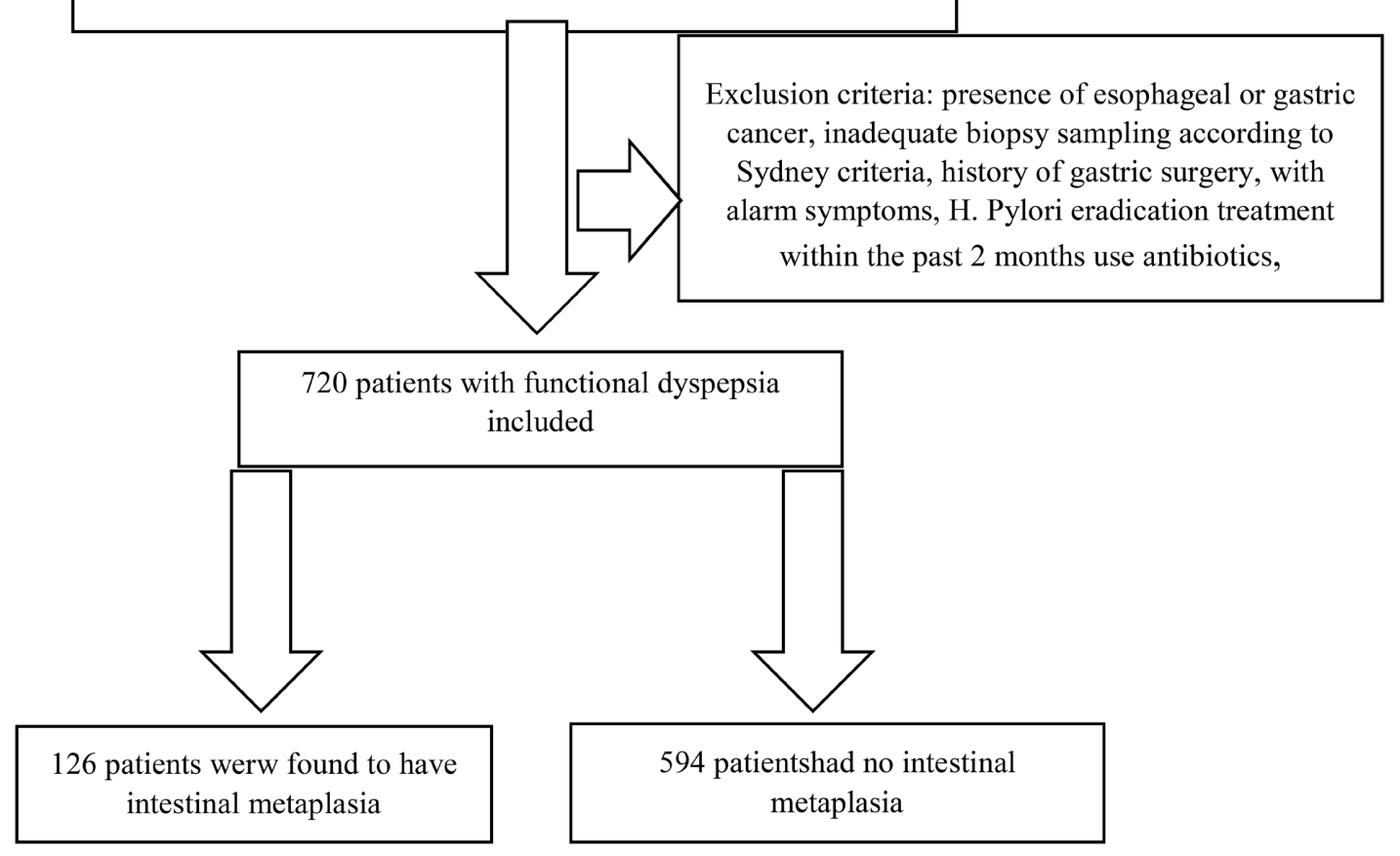

Figure 1. The study design.

was also checked via Giemsa staining. Association between HP infection, chronic proton pump inhibitor (PPI) use exceeding 3 months, and intestinal metaplasia was evaluated.

The study was carried out with the approval of the Institutional Ethical Review Board of Celal Bayar University Medical Center (approval no: 19858. 29/04/2019). The study protocol conforms to the ethical guidelines of the Declaration of Helsinki.

\section{Statistical analyses}

Statistical tests were performed using the Statistical Package for the Social Sciences (SPSS) version 25.0. The IM prevalence across age groups was compared with Kolmogorov Smirnov test and the chi-square test was used to analyze categorical variables, and a value of $\mathrm{P}<0.05$ was regarded as significant.

\section{Results}

720 patients were included in the study. 126 (17.55\%) patients to have intestinal metaplasia. The mean age was 54.31 years, with a range of 18 to 89 years. Of the 126 patients with intestinal metaplasia, 70 (56\%) were male and 56 were female (44\%) (Table 1).

There were no statistically significant differences between genders. 54 (43\%) of the patients with IM were aged 60 years or older, while 72 (57\%) were less than 60 years of age. There was no significant difference in IM patients aged over 60 and less than 60 years of age $(P>0.05)$.

We investigated the relation of patients with intestinal metaplasia with ana- 
tomical involvement site, PPI use and H. pylori (Table 2).

Intestinal metaplasia was detected at the antrum, corpus, and angulus in $59.94 \%$, $13.47 \%$, and $28.57 \%$ of the cases, respectively. Antral lesions were significantly more prevalent $(\mathrm{P}<0.05)$.

Of the patients with IM, $63.5 \%$ had HP positivity, with a significant association between IM positivity and HP positivity $(\mathrm{P}<0.05)$. There was a significant difference between patients with or without a history of chronic PPI use in terms of the prevelance of intestinal metaplasia $(\mathrm{P}<0.05)$.

Additionally, the distribution of IM in anatomical gastric sites exhibited significant variations (Table 3 ).

Table 1. The relation of IM to age and gender.

\begin{tabular}{cccc}
\hline & $\begin{array}{c}\text { Intestinal metaplasia (+) } \\
\text { n: } 126\end{array}$ & Male (\%)/Female (\%) & $\mathbf{P}$ \\
\hline Age & & & \\
$<60$ & 72 & $38(54) / 34(61)$ & \\
$\geq 60$ & 54 & $70(56) / 22(39)$ & \\
Gender & 126 & & \\
\hline
\end{tabular}

Table 2. Involvement site of intestinal metaplasia and its relationship with PPI and $H$. pylori.

\begin{tabular}{cccccc}
\hline & \multicolumn{5}{c}{ Intestinal Metaplasia } \\
\cline { 2 - 6 } n:126 & $(+)$ & $\%$ & $(-)$ & $\%$ & $\mathrm{P}$ \\
\hline Antrum & 73 & 59.94 & 53 & 40.06 & $<0.05 \approx$ \\
Korpus & 17 & 13.49 & 109 & 86.51 & $<0.05^{*}$ \\
Incisura angularis & 36 & 28.57 & 90 & 71.43 & $<0.05^{\star}$ \\
Helicobacter Pylori (+) (n 126) & 80 & 63.50 & 46 & 36.50 & $<0.05 \approx$ \\
PPI (n 126) & 114 & 90.48 & 12 & 9.52 & $<0.05 \approx$
\end{tabular}

${ }^{\star}$ Intestinal Metaplasia $(-)$ was significantly higher than Intestinal Metaplasia $(+) ; \approx$ Intestinal Metaplasia (+) was significantly higher than Intestinal Metaplasia (-); \& Intestinal Metaplasia (+) was significantly higher than Intestinal Metaplasia (-) in Antrum than Korpus and Insisura Angularis.

Table 3. Distribution of intestinal metaplasia among anatomical sublocations.

\begin{tabular}{|c|c|c|c|c|c|c|c|c|}
\hline & \multicolumn{3}{|c|}{ Antrum (n: 73), } & \multirow[b]{2}{*}{$\mathbf{P}$} & \multicolumn{3}{|c|}{ Korpus (n :17), } & \multirow[b]{2}{*}{$\mathbf{P}$} \\
\hline & $\begin{array}{l}\text { Anterior } \\
\text { Wall (\%), }\end{array}$ & $\begin{array}{l}\text { Posterior } \\
\text { Wall (\%) }\end{array}$ & $\begin{array}{l}\text { The Whole } \\
\text { Wall (\%), }\end{array}$ & & $\begin{array}{c}\text { Leser } \\
\text { Cruvature (\%) }\end{array}$ & $\begin{array}{c}\text { Graeter Cruvature } \\
(\%)\end{array}$ & $\begin{array}{c}\text { The Whole Wall } \\
(\%)\end{array}$ & \\
\hline IM+ & $38(52.05)$ & $10(13.7)$ & $25(34.25)$ & $<0.05^{\star}$ & $1(5.88)$ & $6(35.29)$ & $10(58.83)$ & $<0.05 \approx$ \\
\hline
\end{tabular}

${ }^{\star}$ Intestinal Metaplasia $(+)$ was significantly higher than Intestinal Metaplasia $(-)$ in the anterior wall than the other wall. $\approx$ Intestinal Metaplasia $(+)$ was significantly higher than Intestinal Metaplasia $(-)$ in the whole wall than the other wall. In Korpus and insisura angularis, intestinal Metaplasia (-) was significantly higher than Intestinal Metaplasia (+) and in Antrum, H. Pylori (+) patients and PPI user patients intestinal Metaplasia (+) was significantly higher than Intestinal Metaplasia (-). 


\section{Discussion}

Previous studies have shown a strong association between HP infection, facilitation of IM pathogenesis by HP infection, and the precancerous nature of IM when it is comorbid with HP infection [12].

A high prevalence of IM has been noted in HP-positive patients with gastritis. However, IM prevalence may vary between countries or between different geographical regions within a country. Generally, the differences in prevalence correspond to the incidence of gastric cancer [13].

In a study by Fontham et al., a 4.7-fold incidence of IM was found in HP-positive patients compared to HP-negative subjects, which was consistent with our findings showing a significantly higher prevalence of IM in HP-positive subjects [14].

The reported incidence of IM in the study by Craanen et al. was 10\% and 32\% among patients aged $\leq 50$ years and $>50$ years, respectively, showing an increased risk of IM with age [15]. In our study, 43\% of IM patients were over 60 years of age. Although intestinal metaplasia was seen more in the elderly population, it was not statistically significant.

Seventy-three percent of the patients in Craanen et al's study was positive for HP, confirming the important role of HP in the pathogenesis of IM [15].

Our findings are in line with those reported by Adım et al. and Craanen et al., with a significant difference in IM frequency between HP-positive and HP-negative patients $(\mathrm{P}<0.05)$.

In the study by Genta et al., involving 810,821 patients, $8.4 \%$ of the patients had IM, with an increased likelihood of IM among male subjects and the elderly. They found the rate of IM at the age of 18 as $2 \%$ and over the age of 75 at $14 \%$. The lesser curvature of the antrum has been reported to be more frequently involved in HP colonization [16].

In our study, intestinal metaplasia rates were high in our elderly and male patients

The difference observed between gastric sites in terms of HP colonization frequency may be related to the more rapid contraction wave in the greater curvature compared to the lesser curvature, implying a slower flow of gastric liquid in the lesser curvature leading to a relative stasis of the gastric content and HP colonization [17].

In our study, the pathological examination of the samples obtained from angulus and antrum biopsies are also supportive of this notion.

In the study by Eriksson et al., the overall prevalence of intestinal metaplasia was $19 \%$. Intestinal metaplasia was found most frequently in the antrum and in the angulus [18].

In a study by Akpolat et al., IM was found to be present in $14 \%$ and $44 \%$ of the patients $<50$ and $\geq 50$ years of age, respectively. In that study, patients between 20 and 29 years of age had the lowest rate of IM (11\%), while those between 70 and 79 years of age had the highest rate $(83 \%)$, showing an age-related increase 
in IM incidence [19].

Adim et al. detected the presence of IM in $10.2 \%$ of their patients less than 50 years of age compared to $12.7 \%$ of patients over 50 years of age [17]. Again, in our study, patients aged 50 or over had a significantly higher IM risk compared to younger patients.

In the study by Mao et al., the reported incidence of intestinal metaplasia in gastric angulus, antrum, and lesser curvature, and corpus was $28.5,20.24 \%$, and $25.48 \%$, respectively. Intestinal metaplasia was more severe in the lesser curvature and angulus compared to the greater curvature, and HP-positive patients had more severe inflammation in the lesser curvature [2].

In our study, the respective incidences of intestinal metaplasia in the antrum, angulus, and corpus were $59.94 \%, 28.57 \%$, and $13.49 \%$. The most common site of involvement in the antrum was the anterior wall (52.05\%).

HP eradication has been reported to alleviate gastric atrophy and inflammation, although a delayed improvement of gastric atrophy was also shown in patients who receive treatment with PPIs or other acid blockers [19].

Annibale et al. showed an increased rate of improvement in gastric atrophy through HP eradication in patients with normal serum gastrin levels, although gastric atrophy persisted in those with high serum gastrin [20].

In an experimental mouse model, Chen et al. showed associations of hypergastrinemia with gastric atrophy, metaplasia, and cancer development [21].

Additionally, long-term use of PPIs in patients infected with HP was found to worsen atrophic gastritis in the gastric corpus [22]. In our study, a significant difference in the incidence of intestinal metaplasia was found between patients with a history of chronic use of PPIs vs. those without such use $(\mathrm{P}<0.05)$.

\section{Conclusions}

HP-positive patients with atrophic gastritis should undergo HP eradication to prevent and reduce the likelihood of progression to intestinal metaplasia. Patients for whom long-term PPI treatment is planned should be followed up regarding the development of gastric atrophy and intestinal metaplasia.

Additionally, Sydney grading system should always be followed and biopsy sampling from a minimum of 5 sites should be performed in the follow-up of patients with intestinal metaplasia.

\section{Informed Consent}

Endoscopic approval form was obtained from all our patients before endoscopy. It is stated in this consent form that the data can be used in studies.

\section{Source of Finance}

During this study, no financial or spiritual support was received neither from any pharmaceutical company that has a direct connection with the research subject, nor from a company that provides or produces medical instruments and 
materials which may negatively affect the evaluation process of this study.

\section{Authorship Contributions}

Idea/Concept: Tahir Buran, Elmas Kasap; Design: Tahir Buran; Control/ Supervision: Tahir Buran, Elmas Kasap; Data Collection and/or Processing: Tahir Buran, Elmas Kasap; Analysis and/or Interpretation: Tahir Buran, Semin Ayhan; Literature Review; Tahir Buran; Writing the Article: Tahir Buran; Critical Review: Elmas Kasap, Semin Ayhan, Tahir Buran; References and Funding:Tahir Buran; Materials: Tahir Buran, Elmas Kasap.

English language check and correction. Manuscript has been "spellchecked".

\section{Conflicts of Interest}

The authors declare no conflicts of interest.

\section{References}

[1] Ferlay, J., Soerjomataram, I., Dikshit, R., et al. (2015) Cancer Incidence and Mortality Worldwide: Sources, Methods, and Major Patterns in GLOBOCAN 2012. International Journal of Cancer, 136, E359-E386. https://doi.org/10.1002/ijc.29210

[2] Correa, P. (1992) Human Gastric Carcinogenesis: A Multistep and Multifactorial Process-First American Cancer Society Award Lecture on Cancer Epidemiology and Prevention. Cancer Research, 52, 6735-6740.

[3] Mao, X.Y., Xu, S.F., Liu, Q., et al. (2016) Anatomical Predilection of Intestinal Metaplasia Based on 78,335 Endoscopic Cases. Saudi Journal of Gastroenterology, 22, 154-160.

[4] Gomez, J.M. and Wang, A.Y. (2014) Gastric Intestinal Metaplasia and Early Gastric Cancer in the West: A Changing Paradigm. Gastroenterology \& Hepatology, 10, 369-378.

[5] Nunes, P.P., Libânio, D., Pinto, R.M., et al. (2019) Management of Epithelial Precancerous Conditions and Lesions in the Stomach (MAPS II): European Society of Gastrointestinal Endoscopy (ESGE), European Helicobacter and Microbiota Study Group (EHMSG), European Society of Pathology (ESP), and Sociedade Portuguesa de Endoscopia Digestiva (SPED) Guideline Update 2019. Endoscopy, 51, 365-388. https://doi.org/10.1055/a-0859-1883

[6] Fukase, K., Kato, M., Kikuchi, S., et al. (2008) Effect of Eradication of Helicobacter pylori on the Incidence of Metachronous Gastric Carcinoma after Endoscopic Resection of Early Gastric Cancer: An Open-Label, Randomised Controlled Trial. The Lancet, 372, 392-397. https://doi.org/10.1016/S0140-6736(08)61159-9

[7] Kong, Y.J., Yi, H.G., Dai, J.C., et al. (2014) Histological Changes of Gastric Mucosa after Helicobacter pylori Eradication: A Systematic Review and Meta-Analysis. World Journal of Gastroenterology, 20, 5903-5911. https://doi.org/10.3748/wjg.v20.i19.5903

[8] Waldum, H.L. and Fossmark, R. (2018) Types of Gastric Carcinomas. International Journal of Molecular Sciences, 19, Article No. 4109. https://doi.org/10.3390/ijms19124109

[9] Cheung, K.S., Chan, E.W., Wong, A.Y.S., et al. (2018) Long-Term Proton Pump Inhibitors and Risk of Gastric Cancer Development after Treatment for Helicobacter pylori: A Population-Based Study. Gut, 67, 28-35. 
https://doi.org/10.1136/gutjnl-2017-314605

[10] Niikura, R., Hayakawa, Y., Hirata, Y., et al. (2018) Long-Term Proton Pump Inhibitor Use Is a Risk Factor of Gastric Cancer after Treatment for Helicobacter pylori: Retrospective Cohort Analysis. Gut, 67, 1908-1910.

https://doi.org/10.1136/gutjnl-2017-315710

[11] Bray, F., Ferlay, J., Soerjomataram, I., et al. (2018) Global Cancer Statistics 2018: GLOBOCAN Estimates of Incidence and Mortality Worldwide for 36 Cancers in 185 Countries. CA: A Cancer Journal for Clinicians, 68, 394-424. https://doi.org/10.3322/caac.21492

[12] Craanen, M.E., Blok, P., Dekker, W., et al. (1992) Subtypes of Intestinal Metaplasia and Helicobacter pylori. Gut, 33, 597-600. https://doi.org/10.1136/gut.33.5.597

[13] Fraser, A.G., Peng, S. and Jass, J.R. (1998) Intestinal Metaplasia Subtypes and Helicobacter pylori Infection. A Comparison of Ethnic Groups in New Zealand. Journal of Gastroenterology and Hepatology, 13, 560-565. https://doi.org/10.1111/j.1440-1746.1998.tb00690.x

[14] Fontham, E.T., Ruiz, B., Perez, A., Hunter, F., et al. (1995) Determinants of Helicobacter pylori Infection and Chronic Gastritis. American Journal of Gastroenterology, 90, 1094-1101.

[15] Craanen, D.W., Blok, P., Ferwerda, J., et al. (1992) Intestinal Metaplasia and Helicobacter pylori: An Endoscopic Bioptic Study of the Gastric Antrum. Gut, 33, 6-20. https://doi.org/10.1136/gut.33.1.16

[16] Genta, R.M. and Sonnenberg, A. (2015) Characteristics of the Gastric Mucosa in Patients with Intestinal Metaplasia. The American Journal of Surgical Pathology, 39, 700-704. https://doi.org/10.1097/PAS.0000000000000384

[17] Imai, Y., Kobayashi, I., Ishida, S., et al. (2013) Antral Recirculation in the Stomach during Gastric Mixing. American Journal of Physiology: Gastrointestinal and Liver Physiology, 304, G536-G542. https://doi.org/10.1152/ajpgi.00350.2012

[18] Erikssona, N.K., Kärkkäinenb, P.A., Färkkiläa, M.A., et al. (2008) Prevalence and Distribution of Gastric Intestinal Metaplasia and Its Subtypes. Digestive and Liver Disease, 40, 355-360.

[19] Niikura, R., Hayakawa, Y., Hirata, Y., et al. (2019) The Reduction in Gastric Atrophy after Helicobacter pylori Eradication Is Reduced by Treatment with Inhibitors of Gastric Acid Secretion. International Journal of Molecular Sciences, 20, Article No. 913. https://doi.org/10.3390/ijms20081913

[20] Annibale, B., Di Giulio, E., Caruana, P., et al. (2002) The Long-Term Effects of Cure of Helicobacter pylori Infection on Patients with Atrophic Body Gastritis. Alimentary Pharmacology \& Therapeutics, 16, 1723-1731. https://doi.org/10.1046/j.1365-2036.2002.01336.x

[21] Chen, D., Zhao, C.M., Dockray, G.J., et al. (2000) Glycine-Extended Gastrin Synergizes with Gastrin 17 to Stimulate Acid Secretion in Gastrin-Deficient Mice. Gastroenterology, 119, 756-765. https://doi.org/10.1053/gast.2000.16480

[22] Kuipers, E.J., Uyterlinde, A.M., Pena, A.S., et al. (1995) Increase of Helicobacter pylori-Associated Corpus Gastritis during Acid Suppressive Therapy: Implications for Long-Term Safety. American Journal of Gastroenterology, 90, 1401-1406. 